\title{
Reflets
}

Revue d'intervention sociale et communautaire

\section{Intervention communautaire et développement des communautés}

\section{Denis Bourque}

Volume 18, numéro 1, printemps 2012

L'intervention communautaire chez les francophones minoritaires : des pratiques à découvrir

URI : https://id.erudit.org/iderudit/1012331ar

DOI : https://doi.org/10.7202/1012331ar

Aller au sommaire du numéro

Éditeur(s)

Reflets, Revue d'intervention sociale et communautaire

ISSN

1203-4576 (imprimé)

1712-8498 (numérique)

Découvrir la revue

Citer cet article

Bourque, D. (2012). Intervention communautaire et développement des communautés. Reflets, 18(1), 40-60. https://doi.org/10.7202/1012331ar
Résumé de l'article

L'intervention communautaire est liée aux transformations des politiques publiques, des mouvements sociaux et plus largement à celles des enjeux sociaux. La nouvelle action publique ainsi que des initiatives privées basées sur la concertation et la mobilisation des communautés interpellent les intervenants communautaires dans leur pratique et leur éthique. La multiplication des agents de développement des communautés et l'apparition du développement de territoire invitent les intervenants communautaires à décloisonner leur pratique et à relever des défis d'un type nouveau afin de mieux soutenir les systèmes d'action collective locale ciblant les enjeux transversaux autour d'un projet de développement intégré.
Tous droits réservés @ Reflets, Revue d’intervention sociale et communautaire, 2012
Ce document est protégé par la loi sur le droit d'auteur. L'utilisation des services d'Érudit (y compris la reproduction) est assujettie à sa politique d'utilisation que vous pouvez consulter en ligne. 


\section{Intervention communautaire et développement des communautés}

\section{Denis Bourque}

Professeur, Département de travail social, Université du Québec en Outaouais et titulaire de la Chaire de recherche du Canada en organisation communautaire

\section{Résumé}

L'intervention communautaire est liée aux transformations des politiques publiques, des mouvements sociaux et plus largement à celles des enjeux sociaux. La nouvelle action publique ${ }^{1}$ ainsi que des initiatives privées basées sur la concertation et la mobilisation des communautés interpellent les intervenants communautaires dans leur pratique et leur éthique. La multiplication des agents de développement des communautés et l'apparition du développement de territoire invitent les intervenants communautaires à décloisonner leur pratique et à relever des défis d'un type nouveau afin de mieux soutenir les systèmes d'action collective locale ciblant les enjeux transversaux autour d'un projet de développement intégré.

Mots clés : Intervention communautaire, action collective, développement des communautés, développement de territoire, organisation communautaire, agents de développement.

\section{Abstract}

Community intervention is related to the changes in public policy, social movements and more widely to those social issues. New Public Management and private initiatives based on consultation and community mobilization challenge community 
organizers in their practice and ethics. The proliferation of community development workers and the emergence of territory development invite community practitioners to open up their practice and challenges of a new type to better support systems for collective action targeting local issues around cross-cutting an integrated development project.

Key words : Community intervention, collective action, community development, territory development, community organization, community practice, community practitioners.

\section{Introduction}

L'intervention communautaire se déploie au Québec et au Canada depuis plus de cinquante ans. Elle fait référence au travail militant ou professionnel d'intervenants qui visent à influencer l'action collective et le changement social pour contribuer au développement de la société et des groupes qui la composent. Issue des mouvements progressistes des années d'après-guerre - syndical, coopératif, religieux de gauche —, l'intervention communautaire constitue aujourd'hui une profession présente dans le secteur public et communautaire, dans les domaines tels que la santé et les services sociaux, le développement rural, le logement, la famille, la jeunesse, la revitalisation urbaine ou la lutte à la pauvreté (Favreau, 2009).

Dans cet article, nous chercherons à retracer le parcours de l'intervention communautaire telle qu'elle se pratique, particulièrement au Québec. Nous cernerons les formes contemporaines de cette pratique ainsi que les conditions institutionnelles, administratives et politiques qui la façonnent sans jamais la déterminer complètement. Nous nous interrogerons aussi sur les défis et les enjeux éthiques qui confrontent les praticiens de l'intervention communautaire et qui ont un impact sur le sens même du développement des communautés auprès desquelles ils interviennent. 


\section{Trajectoire de l'intervention communautaire}

\section{"L'intervention} communautaire se professionnalise (Bourque, 1997) et est reconnue comme l'une des méthodes majeures du travail social parce qu'elle mise sur l'action collective et parce qu'elle possède un corpus de connaissances, des approches ainsi que des méthodes spécifiques. "
L'intervention collective ou communautaire existait avant la mise en place de l'État-Providence et du réseau de services publics au Canada. Les Églises, les syndicats et certains milieux coopératifs ou de gauche ont mis en place, avant les années 1960, des interventions de soutien et de développement de l'action collective autour d'enjeux sociaux touchant les conditions et les milieux de vie des classes populaires. Même si ces initiatives étaient fortement teintées de prosélytisme, les interventions menées cherchaient à structurer et à rendre plus efficace l'action collective. Il faudra attendre la génération des premiers diplômés universitaires en sciences sociales et en service social pour que l'intervention collective soit portée par une éthique professionnelle et un corpus de connaissances scientifiques en plus des motivations militantes qui l'ont généralement caractérisée.

Au Québec, l'intervention communautaire, aussi appelée organisation communautaire, est pratiquée dans le service public à partir des années 1970 avec la création des Centre local de services communautaires (CLSC). L'intervention communautaire se professionnalise (Bourque, 1997) et est reconnue comme l'une des méthodes majeures du travail social parce qu'elle mise sur l'action collective et parce qu'elle possède un corpus de connaissances, des approches ainsi que des méthodes spécifiques. Elle se distingue de l'action communautaire qui est synonyme d'action collective et citoyenne, alors que l'intervention communautaire vise à agir sur l'action communautaire sous différentes formes et différents rôles, entre autres, démarrage, soutien, structuration, animation et évaluation. L'action collective ou communautaire existe donc même en l'absence de l'intervention communautaire, mais elle peut grandement bénéficier de l'expertise de cette pratique professionnelle.

L'évolution de l'intervention communautaire est liée aux transformations des politiques publiques, des mouvements sociaux et plus largement à celles des enjeux sociaux. Mais le contexte n'est pas la seule donne à prendre en compte. Il y a 
"... l'intervention communautaire a fait l'objet,... d'une reconceptualisation... autour de quatre approches: approche de l'action sociale ou sociopolitique; approche du développement local; approche socioinstitutionnelle; et approche sociocommunautaire. » également l'action des intervenants qui se donnent des moyens pour développer des pratiques réflexives. Ainsi, depuis 1988, les organisateurs communautaires de Centre de santé et de services sociaux (CSSS) au Québec ont créé une communauté de pratiques qui réunit sur une base volontaire la majorité d'entre eux. Le Regroupement québécois des intervenants et intervenantes en action communautaire en CSSS (RQIIAC) leur permet d'influencer le cadre structurel de leur pratique qui « représente à la fois le contexte d'influence sur les pratiques et leur résultat " (Comeau, et collab., 2008, p. 154). Ainsi, la structuration des pratiques d'intervention communautaire relève aussi des stratégies des intervenants pour faire face aux nouveaux enjeux et défis qui les confrontent.

Longtemps conceptualisée autour de la typologie de Rothman $(1979)^{2}$, l'intervention communautaire a fait l'objet, en 2007, d'une reconceptualisation (Bourque, et collab., 2007) autour de quatre approches : approche de l'action sociale ou sociopolitique; approche du développement local; approche socio-institutionnelle; et approche sociocommunautaire. Cette typologie cherche à refléter l'évolution des pratiques autant dans le secteur public que dans le mouvement communautaire et associatif. Elle a été reprise par le Cadre de référence en organisation communautaire du Regroupement québécois des intervenants et intervenantes en action communautaire (RQIIAC, 2010) qui vise à structurer la pratique d'organisation communautaire en CSSS à partir du point de vue professionnel des intervenants. Les quatre approches de cette typologie peuvent être concomitantes ou se succéder dans une même intervention.

L'action sociale ou approche sociopolitique est une approche de défense et de revendication de droits sociaux. Elle prend appui sur le conflit et la pression pour promouvoir les intérêts de groupes opprimés en opposition aux pouvoirs dominants. L'intervention communautaire s'investit alors dans la mobilisation, la structuration de contre-pouvoirs, l'éducation populaire et le développement d'une conscience citoyenne.

Le développement local est une approche qui vise la résolution de problèmes locaux sur la base d'un processus participatif 
impliquant les acteurs sociaux et les citoyens (Comeau, 2007). Le processus est aussi important que les résultats, car il en favorise la pertinence. Il s'agit généralement d'une concertation entre les acteurs locaux dans le cadre de projets réalisés en mode de coopération consensuelle autour d'enjeux potentiellement conflictuels. L'approche du développement local veut renforcer l'autonomie et la capacité d'appropriation du développement par les communautés locales. L'intervention communautaire y joue des rôles d'analyse du milieu, de liaison, de formation et de soutien à la négociation et à la gestion des conflits.

L'approche socio-institutionnelle cherche à ce que les programmes et services publics adaptent leurs interventions aux personnes, aux groupes et aux communautés concernés afin d'en améliorer l'efficacité. L'approche socio-institutionnelle participative a comme finalité le développement de la capacité d'agir des groupes et des collectivités dans une conception où ils deviennent davantage sujets des programmes et services publics développés par des experts et des institutions et moins objets ou consommateurs passifs de ces derniers. L'intervention communautaire organise et soutient la participation et l'organisation des personnes et des groupes en favorisant l'expression des savoirs, des compétences, des expertises et des intérêts citoyens.

L'approche sociocommunautaire cherche à renforcer les liens sociaux et les solidarités de proximité en redonnant aux personnes du contrôle sur leur environnement immédiat. Elle repose sur la continuité d'intervention depuis le renforcement des compétences personnelles jusqu'à l'exercice de la citoyenneté dans le cadre de services de proximité et de réseaux d'appartenance. L'intervention communautaire y soutient le développement de services et d'activités d'entraide, l'appropriation du pouvoir d'agir — empowerment - individuel et collectif, ainsi que la prise de parole citoyenne.

Les quelque 450 organisateurs et organisatrices communautaires qui pratiquent dans les 95 Centres de santé et de services sociaux du Québec (CSSS) ont recours à ces différentes approches. La recherche de Comeau, et collab. (2008) a montré que les approches les plus utilisées varient d'une époque à l'autre, passant 
d'une prédominance de l'action sociale dans les années 1970 à celle du développement local durant les années 1980 et 1990.Au tournant des années 2000 , l'importance croissante des programmes de santé publique semble faire également plus de place à l'approche socio-institutionnelle, de même que la transformation du réseau sociosanitaire au Québec par l'intégration des CLSC dans les CSSS, sorte de méga-établissements issus de la nouvelle gestion publique, très sensibles au modèle médical (Bourque et Lachapelle, 2010).

\section{Concentration d'approches autour du «Développement des communautés "}

"Ces démarches de concertation ont permis de coordonner des actions, d'augmenter l'efficacité des interventions et de se donner du pouvoir collectivement face aux problèmes locaux et aux pouvoirs centraux."
Dans les années 1980, la crise économique a provoqué un chômage endémique et une dévitalisation de nombreux quartiers urbains et régions rurales, et le développement local prend de l'ampleur (Doucet et Favreau, 1991). Des intervenants — dont des organisateurs communautaires de CLSC - et des leaders communautaires - dont certains élus locaux — ont alors mis sur pied les premières tables de concertation afin de pouvoir mener des actions collectives sur des enjeux qui dépassaient leur capacité d'action respective. Par exemple, en 1984, se réunissent au sein d'une table de concertation des organismes communautaires et des institutions qui fondent le Programme économique de Pointe-Saint-Charles (PEP), première expérience de développement économique communautaire en milieu urbain. Le mouvement du développement économique communautaire qui mena à l'édification de l'économie sociale était lancé (Favreau et Lévesque, 1996). On assista à un phénomène similaire sur des problématiques comme le soutien à domicile ou la santé mentale. Ces démarches de concertation ont permis de coordonner des actions, d'augmenter l'efficacité des interventions et de se donner $\mathrm{du}$ pouvoir collectivement face aux problèmes locaux et aux pouvoirs centraux. Ces pratiques expriment la volonté d'acteurs locaux tant institutionnels que communautaires de participer à la recherche de solutions en vue d'améliorer la réponse aux 
«Au cours de la dernière décennie, on a vu se multiplier les initiatives publiques et privées misant sur la mobilisation des communautés et la concertation pour réaliser des actions arrimées à la complexité croissante des enjeux collectifs..." besoins. On ne se contente pas d'adapter les politiques publiques au niveau local, mais aussi de les modifier ou les promouvoir au niveau national comme dans le cas du programme des Carrefours jeunesse-emploi qui tire son origine d'une concertation et d'une innovation sociale menées à Gatineau dans les années 1980 (Assogba, 2000).

La concertation et la mobilisation des acteurs locaux firent donc graduellement leurs armes au Québec à un point tel que les nouvelles politiques publiques - ou programmes privés comme ceux issus de la Fondation Lucie et André Chagnon - en font maintenant l'un de leurs fondements stratégiques ${ }^{3}$.Au cours de la dernière décennie, on a vu se multiplier les initiatives publiques et privées misant sur la mobilisation des communautés et la concertation pour réaliser des actions arrimées à la complexité croissante des enjeux collectifs dans plusieurs domaines de l'action publique - tels le développement de la main-d'œuvre, la sécurité du revenu, la ruralité, la santé et les services sociaux, la santé publique ou l'éducation - jusqu'à provoquer une sorte d'embouteillage (Lévesque, 2006) ou d'hyperconcertation (Bourque, 2008). Si nous en sommes arrivés là, c'est que nombre de nouvelles politiques publiques et privées ont certes repris la méthodologie du développement local, mais s'en éloignent trop souvent quant au sens et aux finalités. Le développement local est originalement un processus du bas vers le haut - bottomup - visant l'appropriation démocratique par les collectivités de leurs enjeux prioritaires en favorisant l'action collective sur leurs causes. Dans une conception et une logique gestionnaires, la concertation et la mobilisation des communautés deviennent plutôt des moyens permettant l'atteinte des objectifs spécifiques d'un programme en mettant à contribution des ressources locales dans une planification qui demeure centralisée. Sur le terrain, les nouvelles pratiques d'action collective regroupées sous l'appellation de "Développement des communautés » représentent une forme de croisement entre ces deux tendances parce que les collectivités locales ont besoin des programmes publics ou privés pour se développer, et parce que ces programmes ont besoin d'une appropriation par les communautés, car on reconnait de plus en 
"...le développement

des communautés

représente pour

les intervenants

communautaires une

sorte d'hybridation

entre l'approche

de développement

local et l'approche socio-institutionnelle

participative qui "vise le développement de la capacité d'agir des collectivités et des communautés"

\section{"Les intervenants} communautaires sont au cour des processus qui permettent d'établir des rapports de collaboration entre les acteurs et contribuent ainsi à la construction d'un système d'action collective. " plus que l'adaptation des interventions publiques ou privées aux réalités locales et aux populations visées représente un meilleur facteur d'efficacité que la fidélité aux programmes (Bilodeau, et collab., 2004; Bisset, 2008).

En fait, le développement des communautés représente pour les intervenants communautaires une sorte d'hybridation entre l'approche de développement local et l'approche socioinstitutionnelle participative qui "vise le développement de la capacité d'agir des collectivités et des communautés " dans les programmes institutionnels ou privés (Bourque et Lachapelle, 2007, p. 116). Cela suppose que les partenaires — institutionnels, privés et communautaires - sont en mesure de négocier leurs contributions sur des bases de réciprocité. Ces rapports de collaboration ne sont toutefois pas sans tensions entre des logiques divergentes (Gourvil, 2011) qui s'inscrivent dans une dynamique paradoxale. D'une part, les institutions, l'État et certains bailleurs de fonds, s'inscrivant dans une logique descendante (voir Figure 1, tirée de Bourque, 2008), développent des programmes qu'ils souhaitent pris en charge par les communautés locales - tendance participative - ou dont ils font une condition d'accès aux ressources requises - tendance instrumentalisation. Ces programmes relèvent d'une logique exogène. D'autre part, les communautés, selon une logique endogène et ascendante, développent des stratégies et des projets concertés pour lesquels elles mobilisent leurs propres ressources et tentent d'accéder aux ressources externes. Certains acteurs locaux acceptent de définir leur action en fonction des programmes - tendance complémentariste - d'autres revendiquent la possibilité d'agir sur leurs propres bases - tendance autonomiste. Le développement des communautés est l'espace sur lequel se négocient les rapports entre ces logiques. Les intervenants communautaires sont au cœur des processus qui permettent d'établir des rapports de collaboration entre les acteurs et contribuent ainsi à la construction d'un système d'action collective. Le développement des communautés gagne en fait à être un processus "négogène " ${ }^{4}$, c'est-àdire en partie exogène parce qu'influencé par les programmes et le financement en provenance des appareils de planification 
et en partie endogène parce que reposant sur la participation et l'appropriation des acteurs. Ce modèle "négogène " donne prise aux acteurs et aux communautés sur le sens et la finalité de l'action tout en s'inscrivant dans des politiques et des programmes qui lui sont indispensables. Les intervenants communautaires qui soutiennent les pratiques de développement des communautés se situent à l'interface entre les logiques descendantes et ascendantes, certains diront entre l'arbre et l'écorce, mais n'est-ce pas là où se trouve la sève?

Figure 1 - Dynamique paradoxale du développement des communautés

\section{Logique descendante-exogène}

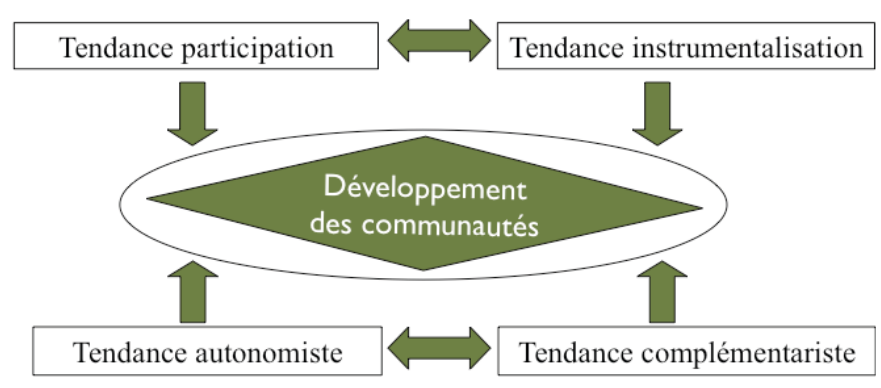

"Le développement des communautés gagne donc à être soutenu par l'expertise d'intervenants communautaires qui y croisent leur maîtrise de l'approche de développement local et de l'approche socio-institutionnelle participative en organisation

\section{Logique ascendante-endogène}

Même si le développement des communautés est une appellation à la mode au Québec, elle est loin d'être une appellation contrôlée. Un peu comme pour la participation ou l'empowerment, on peut y retrouver un large éventail de finalités, d'orientations et de pratiques plus ou moins compatibles (Mercier, 2009). Le développement des communautés gagne donc à être soutenu par l'expertise d'intervenants communautaires qui y croisent leur maitrise de l'approche de développement local et de l'approche socio-institutionnelle participative en organisation communautaire.

communautaire. » 


\section{Multiplication des intervenants communautaires}

"La plupart de ces professions $d u$ développement partagent les caractéristiques de s'exercer sur un même territoire de proximité dans le but de soutenir des projets dans le cadre de missions et de programmes institutionnels en favorisant des rapports de coopération, de concertation ou de partenariat entre les acteurs locaux."
Longtemps pratiquée presque exclusivement par les organisateurs communautaires des CLSC — maintenant CSSS - et des permanents d'organismes communautaires, l'intervention communautaire connait une forte extension en termes d'effectifs et d'employeurs à la faveur du développement des communautés. Plusieurs titres d'emploi désignent les «métiers du développement » (Robitaille, 2007) : organisateur/organisatrice communautaire (450) dans les Centres de santé et de services sociaux; agent de développement rural (180) et agent d'économie sociale (150) dans les Municipalités régionales de comté et les Centres locaux de développement; agent de liaison (80) dans les programmes issus de la Fondation Chagnon ${ }^{5}$; agent de concertation et de développement en nombre indéterminé dans les différentes municipalités et les Conférences régionales des Élus; permanents d'organismes communautaires en nombre indéterminé dans des Corporations de développement communautaire (50 au Québec), des tables de développement social (30 à Montréal). Sans nier les particularités des démarches auxquelles elles renvoient, nous estimons avec Comeau, et collab. (2008) que, derrière la diversité des appellations, ces pratiques se rattachent au corpus commun de l'intervention communautaire. La plupart de ces professions du développement partagent les caractéristiques de s'exercer sur un même territoire de proximité dans le but de soutenir des projets dans le cadre de missions et de programmes institutionnels en favorisant des rapports de coopération, de concertation ou de partenariat entre les acteurs locaux. Ces différents intervenants peuvent œuvrer dans le cadre de mandats étroits comme les agents de liaison de Québec en Forme qui vise à promouvoir les saines habitudes de vie chez les jeunes, ou de mandats larges comme les agents de développement rural qui visent le renforcement de la capacité des territoires à développer et à dynamiser leur communauté locale. Dans tous les cas, l'intervention cible l'action collective et la concertation comme conditions de réalisation de la mission ou du programme qui mandate l'intervenant. Dans ces 
"La réussite des démarches d'action collective et de concertation associées au développement des communautés nécessite que les intervenants communautaires pilotent des processus complexes." domaines, l'action collective et la concertation comportent des exigences de réussite dont les quatre premières sont identifiées par Bilodeau, Chamberland et White (2002) :

1. la mobilisation des acteurs pertinents à la question en jeu;

2. que ces acteurs soient mobilisés sur des choix stratégiques et non seulement techniques ou instrumentaux;

3. qu'ils soient également en position d'influence et de négociation - au-delà de la consultation;

4. que le processus participatif permette de réduire l'inégalité de pouvoir entre les acteurs - dont les intervenants - et favorise la coconception et coconstruction des actions.

Bourque et Lachapelle (2007) ajoutent les quatre facteurs de réussite suivants :

5. la pertinence sociale du programme ou du projet à l'origine de l'action collective et de la concertation;

6. la flexibilité et l'ouverture de l'organisme promoteur ou du bailleur de fonds;

7. l'appropriation créative du programme ou du projet par les acteurs concernés;

8. la constance du soutien organisationnel et professionnel en provenance ou non de l'organisme promoteur.

Enfin, Desjardins (2010) identifie deux derniers facteurs :

9. la crédibilité et l'efficacité de l'action collective et de la concertation entreprises;

10.l'intégration et la priorisation des objectifs issus de l'action collective et de la concertation dans le plan d'action des partenaires concernés.

La réussite des démarches d'action collective et de concertation associées au développement des communautés nécessite que les intervenants communautaires pilotent des processus complexes. Ces processus commandent des compétences spécifiques et surtout font appel à un fort ancrage éthique sur les valeurs de participation et de démocratie. L'identité professionnelle des intervenants communautaires est en jeu. Sont-ils avant tout des agents de programme ou des agents de développement? La notion 
de développement que nous retenons est celle du Conseil de la santé et du bien-être (CSBE) selon lequel "le développement est un processus, une démarche par laquelle une communauté, par voie d'initiatives, tente de maintenir ou d'améliorer, selon les valeurs qu'elle juge prioritaires, les conditions de vie collectives et/ ou individuelles » (2001,p.11). Le sens du développement provient donc fondamentalement des communautés et des acteurs qui les composent. Pour les agents de développement, une telle posture ne va pas sans poser des dilemmes de loyauté ou d'allégeance entre leur employeur qui a sa mission, ses objectifs et ses intérêts, et leurs communautés qui ont à s'approprier leur développement dans un sens qui n'a pas à être déterminé de l'extérieur. Composer avec ces dilemmes n'est pas simple et Savard (2007) propose quelques moyens pour y arriver. Retenons ici un principe de référence : les missions institutionnelles et organisationnelles d'une grande complexité comme améliorer l'état de santé des populations, favoriser le développement des jeunes ou des familles ou encore prévenir le décrochage scolaire ne peuvent être atteintes sans un engagement des personnes, des acteurs et des communautés concernés. La qualité de cet engagement est proportionnelle au

"En d'autres mots, soutenir des processus d'action collective et de concertation qui permettent d'agir sur les causes des problèmes et non seulement sur leurs conséquences ou symptômes..." développement du pouvoir d'agir des acteurs qui se traduit par la capacité d'innover et par la pertinence et la pérennité des actions collectives mises en œuvre. En d'autres mots, soutenir des processus d'action collective et de concertation qui permettent d'agir sur les causes des problèmes et non seulement sur leurs conséquences ou symptômes, qui permettent d'agir sur les conditions et les milieux de vie, qui permettent de mettre à contribution les savoirs et expertises des personnes et groupes concernés, bref, qui visent une prise en main des enjeux collectifs, voilà qui est de nature à contribuer à l'atteinte des missions sociales institutionnelles et organisationnelles.

Malgré tout, l'intervention des agents de développement demeure paradoxale, car même s'ils sont promus par des politiques publiques, les processus locaux de concertation et de développement des communautés sont dissonants par rapport au mode classique d'intervention publique et à certains éléments de la culture administrative issue de la nouvelle gestion publique 
(Bourque, et collab., 2011). Ils sont de nature paradoxale, car tout en s'inscrivant dans la planification institutionnelle et programmatique, ils mettent en mouvement des systèmes d'action collective qui échappent au contrôle étatique ou des bailleurs de fonds dans le cadre de projets communs à développer (Divay, 2009). Pour réussir, ces processus appellent des innovations que les politiques publiques ou les programmes centralisés et spécialisés par problématique ou population cible ne favorisent pas. Des communautés territoriales se sont donc données, ou tentent de se donner, une vision cohérente de développement et des stratégies qui ont permis de coordonner les efforts des acteurs visant le développement territorial intégré (Aubin, 2007; Ulysse et Lesemann, 2007; Moulaert et Nussbaumer, 2008).

\section{Développement de territoire}

"...les forces de la désintégration et l'incohérence des approches institutionnelles peuvent être contrecarrées en plaçant les besoins et l'organisation des groupes exclus au coeur du développement territorial intégré basé sur la satisfaction des besoins essentiels. »
Le développement des communautés se pratique très souvent autour d'une problématique, par exemple la santé mentale, ou d'une population cible comme la jeunesse ou les personnes âgées. Il s'agit ici de communautés d'identité ou d'intérêt auxquelles il faut ajouter les communautés territoriales. Le territoire renvoie à la notion de communauté comprise comme une population habitant un territoire donné caractérisé non seulement comme espace géographique, mais aussi comme espace où les réseaux de relations entre les personnes, les groupes et les institutions constituent un ensemble social organisé. Selon Moulaert et Nussbaumer (2008), les forces de la désintégration et l'incohérence des approches institutionnelles peuvent être contrecarrées en plaçant les besoins et l'organisation des groupes exclus au cœur du développement territorial intégré basé sur la satisfaction des besoins essentiels. Cette satisfaction devrait s'effectuer par la combinaison de plusieurs processus : la révélation de besoins par des mouvements sociaux et par des dynamiques institutionnelles; l'intégration des groupes exclus dans le marché du travail et dans l'organisation des réponses collectives aux besoins locaux; et la participation significative à la consultation et à la prise de 
décision politique sur l'avenir des territoires sur le plan social, économique et environnemental. La communauté locale pourrait par cette voie prendre possession de sa propre gouvernance, et par conséquent placer sa propre dynamique au centre du processus de développement de territoire qui comporte des caractéristiques qui augmentent le potentiel de l'action collective :

1. Il favorise une prise en compte globale des enjeux collectifs en ciblant la communauté dans son ensemble dans ses dimensions sociale, économique, environnementale et culturelle;

2. Il implique nécessairement des actions intersectorielles et transversales puisque les interventions doivent composer avec divers niveaux d'organisation territoriale (local, régional, national, international), diverses missions de l'État (santé, emploi, éducation, etc.), divers groupes sociaux (enfants, jeunes, familles, personnes âgées), diverses problématiques (pauvreté, exclusion, santé mentale, environnement, etc.), divers acteurs sociaux (privé, public, économie sociale);

3. Il contribue, sous certaines conditions, à augmenter le sentiment d'appartenance et d'identité au territoire et constitue un moteur de l'action collective. Comme Klein le mentionne, le développement de territoire réfere à l'accroissement de la capacité de "certaines collectivités d'agir en systèmes, où les compromis entre les acteurs sont largement influencés par l'identité territoriale chez les acteurs " (2006, p. 151).

Pour Caillouette, et collab., le développement de territoire réfere à :

des stratégies, des processus et des dispositifs déployés par les acteurs afin de construire leurs actions selon le passage d'une rationalité individuelle, institutionnelle ou organisationnelle à une "relationnalité " territoriale, et c'est cette activité relationnelle, en croisement de différentes logiques d'action, qui contribue à l'émergence d'un acteur local collectif sujet de son développement. [...] La territorialité est la construction plurielle d'un territoire vécu basée 
"Le développement territorial vise à faire monter d'un cran l'intensité de l'action collective en misant sur une négociation entre les acteurs concernés de façon à susciter et maintenir une vision globale qui touche les enjeux sociaux, économiques et environnementaux d'un milieu. »

\section{"Le développement} de territoire incite les différents agents de développement à décloisonner leur pratique. » sur un processus de mise en lien des acteurs à partir d'une identité territoriale qui contribue à la construction de celle-ci (2007a, p. 13).

Le développement territorial vise à faire monter d'un cran l'intensité de l'action collective en misant sur une négociation entre les acteurs concernés de façon à susciter et maintenir une vision globale qui touche les enjeux sociaux, économiques et environnementaux d'un milieu. Les perspectives de développement durable, d'occupation du territoire, de prise en compte des déterminants sociaux de la santé ou de lutte contre la pauvreté et l'exclusion sociale ont en commun de s'appliquer sur une base territoriale. Elles demandent aux acteurs d'un même territoire de négocier des collaborations essentielles pour mettre en œuvre des projets reposant sur des coopérations de différentes natures susceptibles de coconstruire des arrangements « viables, vivables et équitables » entre les secteurs — social, économie, environnement - et entre les acteurs, et à produire des innovations institutionnelles et professionnelles pour les soutenir (Jacquier, 2009; 2010).

Le développement de territoire incite les différents agents de développement à décloisonner leur pratique. Ils ont à exercer une fonction de liaison entre les démarches de concertation thématique - par problématique et population cible - entre les institutions, entre les organismes citoyens, entre les unes et les autres et avec les expertises issues de la recherche. En tant que processus, la recherche fait mention que les intervenants communautaires devraient exercer un leadership propre à favoriser un fonctionnement efficace alliant à la fois action-réflexion et une connaissance du territoire favorisant la coopération entre les secteurs et les acteurs du développement (Lachapelle, et collab., 2009). Cette action aux frontières des organisations pose des défis qui demandent à être mieux identifiés et repose sur une expertise du territoire dont la nature reste à préciser. En effet, les recherches menées jusqu'à présent sur le développement de territoire ont peu touché l'intervention professionnelle de soutien au système d'action collective locale pour se centrer davantage sur sa dynamique et son ingénierie (Divay, et collab., 2010). Ces 
recherches ont tout de même pointé un certain nombre de conditions favorables et de contraintes avec lesquelles doivent composer les intervenants communautaires (Caillouette, et collab., 2007b; Klein, 2011). Sur le terrain, le développement de territoire s'opère selon deux principes qui cohabitent et se métissent :

1. Celui de la coopération où les enjeux collectifs des territoires sont travaillés par les acteurs locaux et les populations, en interface avec des politiques publiques ou des programmes privés dans un possible rapport conflictuel. On parle ici de territorialité du développement qui, pour se construire, "demande à ce que la coopération locale, horizontale et intersectorielle entre les acteurs ait droit de cité face aux orientations verticales, nationales et sectorielles auxquelles sont par ailleurs soumis ces acteurs " (Caillouette, et collab., 2008, p. 19).

2. Celui de la déconcentration des politiques publiques (Hamzaoui, 2002) où la mobilisation des communautés territoriales est une stratégie de gestion des programmes publics ou privés pour en assurer l'implantation et en augmenter la prégnance. On parle alors d'enveloppement plutôt que de développement de territoire (Bernoux, 2005).

Le développement de territoire apparait donc comme un nouveau champ de pratique pour l'intervention communautaire qui est appelée à puiser dans ses différentes approches et peutêtre à en inventer de nouvelles pour mieux soutenir ces systèmes

"Le développement de territoire étant fortement associé au déploiement de politiques et programmes publics et privés, il interpelle l'approche socioinstitutionnelle en intervention communautaire. " d'action collective locale. Le développement de territoire étant fortement associé au déploiement de politiques et programmes publics et privés, il interpelle l'approche socio-institutionnelle en intervention communautaire. Mais ce développement de territoire n'ayant de sens que par son appropriation par les collectivités concernées, il doit faire appel et reposer principalement sur l'approche de développement local qui en devient le noyau dur autour duquel gravitent les apports des autres approches dont l'approche sociocommunautaire compte tenu des enjeux actuels de cohésion et d'inclusion sociale. Le développement de territoire s'inscrit aussi dans des rapports sociaux conflictuels qui peuvent 
opposer des intérêts particuliers entre eux ou au bien commun. L'action collective en développement de territoire doit aussi prendre la forme de la contestation de politiques publiques ou de pratiques privées inacceptables comme celle de l'exploitation des gaz de schiste dans sa forme actuelle, ce qui indique comment l'approche de l'action sociale demeure aujourd'hui toujours nécessaire et pertinente. Enfin, la pérennité du développement de territoire passe par un certain nombre d'orientations stratégiques de la part des intervenants communautaires et des agents de développement dont celle de développer des organisations autonomes des pouvoirs publics et privés, organisations démocratiques et basées sur la participation citoyenne.

\section{Conclusion}

Le développement des communautés - d'identité, d'intérêt, de territoire - donne de meilleurs résultats sous certaines conditions que les intervenants communautaires ont appris à mettre en œuvre et à maitriser dont la qualité du processus participatif et démocratique, une prise en compte globale dans l'analyse des problématiques avec leurs dimensions sociales, économiques, écologiques et politiques, la qualité du travail de liaison et de médiation entre les cultures des partenaires intersectoriels, l'activation d'une interface productive entre les logiques descendante - top-down - et ascendante - bottomup - le soutien au leadership partagé, la gestion des conflits, etc. (Lachapelle, et collab., 2009). Il y a là une expertise qui mérite d'être mise à contribution pour dépasser les limites actuelles de pratiques qui instrumentalisent la concertation et le développement des communautés et en compromettent l'efficacité et même l'avenir. Le courant actuel favorable au développement des communautés ne résistera pas au cul-de-sac que représente à terme une "nouvelle action publique " (Gaudin, 2007) qui cherche à activer les dynamiques locales et l'action concertée avec des règles du jeu et des logiques contre-productives. Pour produire des réponses adaptées et innovantes quant aux enjeux 
d'aujourd'hui, la concertation doit dépasser la simple gestion de programmes. Elle doit aussi dépasser la fragmentation sectorielle des problèmes collectifs - selon les missions ministérielles ou les objectifs de programmes privés - pour travailler les territoires dans leurs enjeux globaux et transversaux autour d'un projet de développement intégré et durable.

En l'absence d'une volonté politique nationale pour mieux orienter et coordonner l'action publique en ce domaine, des acteurs régionaux et locaux mettent pied à terre et décident de réfléchir collectivement. Les intervenants communautaires peuvent et doivent contribuer à ces exercices même s'ils n'y sont pas spontanément invités. Les conditions d'une meilleure articulation entre les politiques publiques ou les programmes privés et les pratiques de concertation et de développement des communautés sont encore à identifier. Cela ne pourra être fait sans l'apport des intervenants communautaires et autres agents de développement.

\section{Notes}

1 La Nouvelle Action Publique se caractérise par un recours aux arrangements contractuels pour le déploiement des politiques publiques qui « ouvrent l'action publique à des négociations avec les pouvoirs locaux » (Gaudin, 2006, p. 26) et offre donc des potentialités aux initiatives locales mais avec des contraintes qui peuvent en détourner le sens.

2 La typologie de l'organisation communautaire de Rothman (1979) comprend trois approches : l'action sociale, le développement local et le planning social. Comme le notent Doucet et Favreau (1991), le planning social peut appartenir à l'organisation communautaire ou à l'administration sociale, ce qui a toujours posé une difficulté conceptuelle à inclure cette approche dans les modèles de base de l'organisation communautaire. Le planning social se définit comme un processus fondé sur le recours aux experts pour structurer des programmes et des services — publics ou communautaires — visant la résolution de problèmes communautaires prioritaires. La mise sur pied de services de première ligne s'adressant à des populations cibles constitue difficilement en soi une pratique d'organisation communautaire. Pour ce faire, il faut que ces services s'appuient sur la participation des populations concernées et favorisent l'empowerment des collectivités, ce qui n'est pas une exigence explicite dans le modèle de planning social. Contrairement au planning social, l'approche socio-institutionnelle place la participation significative des personnes et des collectivités concernées au cœur de ses pratiques.

3 En 2003, le Programme national de santé publique fait du développement des communautés une de ses stratégies de base (MSSS, 2003). 
4 L'expression est de Claudine Papin de la Coopérative Tandem. Elle a été entendue lors de la Rencontre régionale du développement local tenue à Gatineau le 6 juin 2003.

5 On pense, entre autres, à Québec en Forme, Avenir d'enfants, Réunir Réussir, Proches Aidants.

\section{Bibliographie}

ASSOGBA, Yao (2000). Insertion des jeunes, organisation communautaire et société. L'expérience fondatrice des CJE, Québec, Presses de l'Université du Québec, 151 p.

AUBIN, Jean-François (2007). «Les démarches de revitalisation intégrée, un nouveau défi pour le travail social », Intervention, $\mathrm{N}^{\circ} 126$, p. 127-135.

BERNOUX, Jean-François (2005). Mettre en œuvre le développement social territorial, Méthodologie, outils, pratiques, Paris, Dunod, $201 \mathrm{p}$.

BILODEAU, Angèle, Claire CHAMBERLAND et Deena WHITE (2002). «L'innovation sociale, une condition pour accroître la qualité de l'action en partenariat dans le champ de la santé publique ", Revue canadienne d'évaluation de programmes, Vol. 17, No 2, p. 59-88.

BILODEAU, Angèle, et collab. (2004). "L'exigence démocratique de la planification participative : le cas de la santé publique au Québec ", Nouvelles Pratiques sociales, Vol. 17, № 1, p. 50-65.

BISSET, Sherri (2008). Health program implementation, a socio-technical networking process. A case study of a school-based nutrition intervention, [thèse de doctorat], Montréal, Département de médecine sociale et préventive, Université de Montréal.

BOURQUE, Denis (1997). «Trajectoire de l'organisation communautaire professionnelle », Nouvelles pratiques sociales, Vol. 10, № 1, 1997, p. 59-70.

BOURQUE, Denis, et collab. (dirs.) (2007). Organisation communautaire, fondements, approches et champs de pratique, Québec, Presses de l'Université du Québec, 560 p.

BOURQUE, Denis, et René LACHAPELLE (2007). "L'approche socio-institutionnelle en organisation communautaire ", dans Denis Bourque, et collab. (dir.), L'organisation communautaire, fondements, approches et champs de pratique, Québec, Presses de l'Université du Québec, p. 101-117.

BOURQUE, Denis, et René LACHAPELLE (2010). Service public, participation et citoyenneté : L'organisation communautaire en CSSS, Québec, Presses de l'Université du Québec, 165 p.

BOURQUE, Denis (2008). Concertation et partenariat : entre levier et piège du développement des communautés, Québec, collection Initiatives, Presses de l'Université du Québec, 152 p.

BOURQUE, Denis, et collab. (2010). Les effets de la création des CSSS sur les pratiques partenariales, psychosociales et communautaires, Rapport de recherche, Chaire de recherche du Canada en organisation communautaire, UQO, Cahier No 1004, 175 p.

CAILlOUETTE, Jacques, et collab. (2007a). Territorialité, action publique et développement des communautés, Économie et Solidarités, Québec : CIRIEC-Canada, Université du Québec en Outaouais, Presses de l’Université du Québec,Vol. 38, № 1, p. 8-23.

CAILLOUETTE, Jacques, et collab. (2007b). «Le développement des communautés locales comme enjeu de renouvellement de l'intervention publique communautaire ", Intervention, $\mathrm{N}^{\circ} 126$, p. 31-41.

CAILlOUETTE, Jacques, et collab. (2008). Étude de pratiques innovantes de développement des communautés dans les 7 CSSS de l'Estrie. Analyse transversale de 7 études de cas, CSSS-IUGS de Sherbrooke, $124 \mathrm{p}$. 
COMEAU, Yvan (2007). "L'approche de développement local en organisation communautaire ", dans Denis Bourque, et collab. (dir.), L'organisation communautaire, fondements, approches et champs de pratique, Québec, Presses de l'Université du Québec, p. 59-79.

COMEAU,Yvan, et collab. (2008). L'organisation communautaire en mutation. Études sur la structuration d'une profession du social, Québec, Presses de l'Université Laval, 194 p.

CONSEIL DE LA SANTÉ ET DU BIEN-ÊTRE (2001). L'appropriation par les communautés de leur développement, Québec.

DESJARDINS, Denise (2010). Le soutien professionnel au projet écoles et milieux en santé comme stratégie de développement des communautés, Denis Bourque (dir.), Chaire de recherche du Canada en organisation communautaire, UQO, Cahier No 1001.

DOUCET, Laval, et Louis FAVREAU (1991). Théorie et pratiques en organisation communautaire, Québec, Presses de l'Université du Québec, 468 p.

DIVAY, Gérard (2009). "La réingénierie civique des services décentralisés dans un État stratège », dans Louis Côté, Benoît Lévesque et Guy Morneau (dirs.), État stratège et participation citoyenne, Québec, Presses de l'Université du Québec, p. 165-205.

DIVAY, Gérard, et collab. (2010). Évaluation des processus et développement de connaissances sur la revitalisation urbaine intégrée dans cinq territoires de la ville de Montréal, (Annexe), Rapport complet présenté au MESSS, Centre de recherche et d'expertise en évaluation (CREXE), ÉNAP.

FAVREAU, Louis, et Benoît LÉVESQUE (1996). Développement économique communautaire Économie sociale et intervention, Sainte-Foy, Presses de l'Université du Québec, 230 p.

FAVREAU, Louis (2009). Organisation communautaire en CLSC et associations citoyennes : un avenir incertain, dans Carnet de Louis Favreau Chaire de recherche en développement des collectivités (CRDC), réf. du 18 février 2012, http://jupiter.uqo.ca/ries2001/carnet/spip.php?article36.

GAUDIN, Jean-Pierre (2006). « La contractualisation des politiques et la nouvelle action publique », dans Yves Luchaire (dir.), Collectivités territoriales et gouvernance contractuelle, Paris, L'Harmattan, $218 \mathrm{p}$.

GOURVIL, Jean-Marie (2011) «Formation et territoire : l'héritage de l'époque moderne en question ", dans Henry Noguès, Marc Rouzeau et Yvette Molina (dirs.), Le travail social et ses formations à l'épreuve des territoires, UNAFORIS, Presses de l'EHESP, Coll. Politiques et interventions sociales, $288 \mathrm{p}$.

HAMZAOUI, Medjed (2002). Le travail social territorialisé, Bruxelles, Éditions de l'Université de Bruxelles, $189 \mathrm{p}$.

JACQUIER, Claude (2009). "Politique de la ville et développement soutenable : recyclage des communautés-territoires et coopération entre les acteurs de la ville ». DIV SG-CIV, Revue électronique Demain la Ville, réf. du 18 février 2012, http://www.prisme-asso.org/spip. php?article 4028

JACQUIER, Claude (2010). Défis et opportunités pour la gouvernance multiniveau en Europe. Villes et régions urbaines au cœur d'un développement soutenable fondé sur la coopération, Multilevel Urban Governance Conference, Présidence belge de l'Union européenne, Liège, 1er et 2 janvier, keynote speaker, 15 pages, réf. du 18 février 2012, http://halshs.archives-ouvertes. fr/halshs-00561062 
KLEIN, Juan-Luis (2006). "Développement local et initiative locale. Une perspective d'analyse et d'intervention ", dans Marielle Tremblay, Pierre-André Tremblay et Suzanne Tremblay (dirs.), Le développement social. Un enjeu pour l'économie sociale, Sainte-Foy, Presses de l'Université du Québec, p. 150-161.

KLEIN, Juan-Luis (2011). "Économie sociale et territoire en contexte de mondialisation. Le développement de l'initiative locale ", dans Guy Bellemare et Juan-Luis Klein (dirs.) (2011). Innovation sociale et territoire. Convergences théoriques et pratiques, Québec, Presses de l'Université du Québec, p.175-194.

LACHAPELLE, René, BOURQUE, Denis et Dominique FOISY (2009). Apports de l'organisation communautaire en CSSS aux nouvelles infrastructures communautaires de développement des communautés, [avec la collaboration de Julie Sénéchal], Chaire de recherche du Canada en organisation communautaire, UQO, Cahier $\mathrm{N}^{\circ} 0902$.

LÉVESQUE, Benoît (2006). Une gouvernance partagée et un partenariat institutionnalisé pour la prise en charge des services d'intérêt général, Cahier C-13-2006, CRISES, UQAM.

MERCIER, Clément, (2009). Participation citoyenne et développement des communautés au Québec: enjeux, défis et conditions d'actualisation, Denis Bourque (dir.), [avec la collaboration de Lise StGermain], Cahier de l'ARUC-Innovation sociale et développement des communautés, Série Documentation et diffusion, $\mathrm{N}^{\circ} 8$, UQO.

MOULAERT, Frank, et Jacques NUSSBAUMER (2008). La logique sociale du développement territorial, Québec, Presses de l'Université du Québec, 153 p.

MINISTÈRE DE LA SANTÉ ET DES SERVICES SOCIAUX (2003). Programme national de santé publique 2003-2012, Québec, Direction générale de la santé publique, ministère de la Santé et des Services sociaux.

ROBITAILLE, Martin (2007). Les métiers du développement local et régional au Québec : l'émergence de nouvelles compétences, Université du Québec en Outaouais : Chaire de recherche du Canada en développement des collectivités (CRDC) - Centre de recherche sur le développement territorial (CRDT- UQO), Recherches, Cahier No 9.

ROTHMAN, Jack (1979). «Three models of community organization practice, their mixing and phasing ", dans Fred M. Cox, et collab., Strategies of Community Organization, Itasca, Illinois, Peacok Publishers, $561 \mathrm{p}$.

RQIIAC (2010). Pratiques d'organisation communautaire en CSSS Cadre de référence du RQIIAC, Québec, Presses de l'Université du Québec.

SAVARD, Sébastien (2007). "Conflits de valeurs et organisation communautaire au Québec ", dans Denis Bourque, et collab. (dirs.), L'organisation communautaire, fondements, approches et champs de pratique, Québec, Presses de l'Université du Québec, p. 281-296.

ULYSSE, Pierre-Joseph, et Frédéric LESEMANN (2007). Lutte contre la pauvreté, territorialité et développement social Le cas de Trois-Rivières, Québec, Presses de l’Université du Québec, 154 p. 